\title{
EDITORIAL
}

\section{Reproductive Immunology Issue 2: Cellular and Molecular Biology}

\author{
Guest Editor B Anne Croy
}

Cellular \& Molecular Immunology (2014) 11, 503-505; doi:10.1038/cmi.2014.98; published online 29 September 2014

In Issue 1 of this special topic series on Reproductive Immunology (September 2014 issue of Cellular and Molecular Immunology, Vol. 11, No. 5), immune cell populations in the reproductive tracts of males and non-pregnant females were discussed. Emphasis was placed on endocrine regulation of the specialized cells that populate these mucosal tissues. ${ }^{1-3}$ The dynamic changes in the uterine immune system induced over the course of a healthy pregnancy were also discussed. ${ }^{2,4-7}$ In Issue 2 of this special topic mini-series, the contributed papers are more clinically oriented and address current understanding of the immunological aspects of common pathologies that complicate human and livestock reproduction. Rodent models that provide key translational insights are blended with the clinical information. The issues addressed are broad. They range from infertility, through pregnancy diseases and loss, preterm labour and roles of the clinical diagnostic laboratory in supporting advances that will translate into improved clinical care and human health. In the contemporary framework, immune system promotion of reproductive success (immunotrophism) is strongly supported, while immune systeminduced complications and failures of reproduction provide key opportunities to understand the disruption of homeostasis. Because each mammal must experience pregnancy during fetal life, and because a healthy gestation sets a pattern for long term postnatal health, ${ }^{8}$ the influences of the maternal immune system on pregnancy and perinatal outcomes are fascinating in themselves. Maternal immune influences on pregnancy, however, also shape clinical outcomes and patient care and need to be more widely understood and appreciated. The international contributors to this issue hope that readers will find these topics of interest and that their articles will stimulate further exploration of pregnancy-related immunology by a wider community of well-trained and experienced immunologists.

Issue 2 of this mini-series begins with a discussion of female infertility due to ovarian autoimmune disease. Warren and his

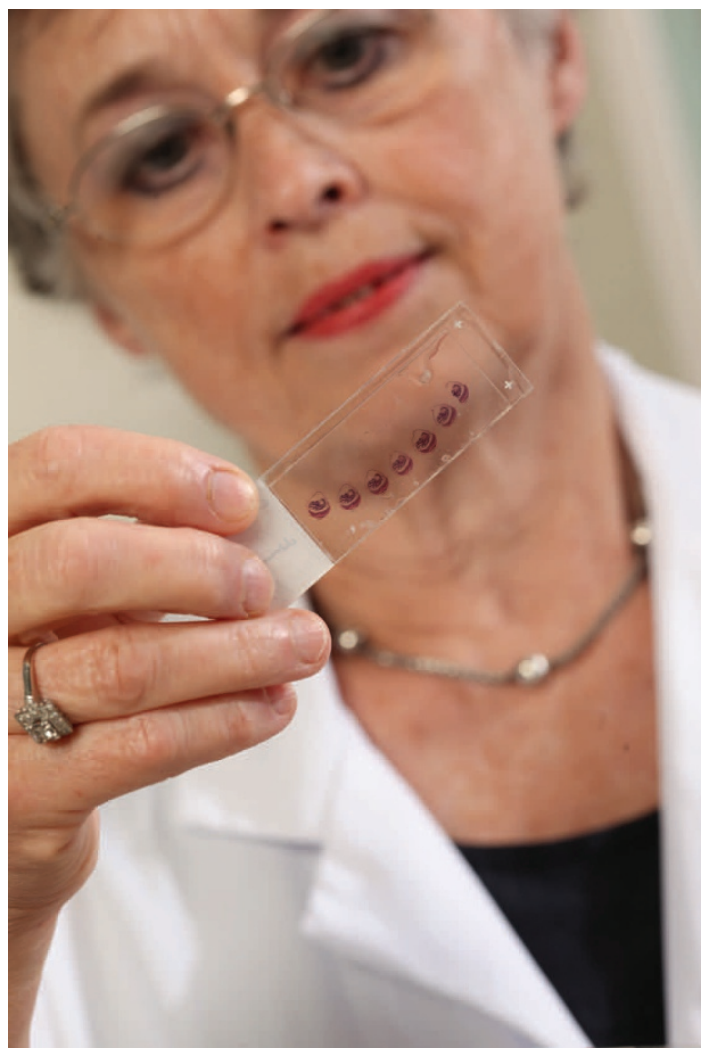

colleagues $^{9}$ describe this component of many autoimmune diseases and focus on thymic regulation of autoimmune $\mathrm{T}$ cells and on antigens induced by the transcription factor AIRE in medullary thymic epithelial cells that are also found in the reproductive tracts of mice and humans of each sex.

Angiogenesis in the maternal endometrial layer of the uterus is a critically important event at implantation sites. In rodents and women, specialized immune cells contribute to the vascular preparation of an adequate 'placental bed'. Lima and her 
colleagues ${ }^{10}$ review the processes of endometrial decidualization and decidual angiogenesis in early pregnancy and its importance to pregnancy success. They also emphasize the importance of proper vascular changes during early pregnancy for support of appropriate maternal and fetal cardiac changes during pregnancy. Variations in these processes may contribute to the gains in postpartum cardiovascular disease risks of women and their offspring. ${ }^{11}$

Many species do not form a hemochorial placenta and do not have invasive trophoblast cells. The commercially important pig is an example of a species with a noninvasive epitheliochorial placenta. Immune-promoted endometrial angiogenesis remains critical for determination of implantation site viability or death in species with superficial placentation. Regulation of immune promoted angiogenesis and its role in spontaneous porcine fetal loss are discussed by Bidarimath and his colleagues ${ }^{12}$ with an emphasis on regulation of the immune cells and of placental trophoblast cells by microRNAs.

MicroRNAs are not only found in cells but also in circulating exosomes. Placenta is a known, abundant but also transient source of vesicles that include exosomes. Tannetta and her colleagues ${ }^{13}$ review extracellular vesicles of the male and female reproductive tracts and their potential functions as well as placentally-derived materials that were originally considered to be 'debris' or 'junk'. The relationship of these materials to pregnancy success through maternal immune cell regulation and to pregnancy complications such as preeclampsia and preterm labour are highlights of the Tannetta group's communication.

Humans are a relatively infertile species and recurrent pregnancy loss (abbreviated RPL and also called recurrent spontaneous abortion (RSA)) in specific women or in specific couples is a common but incompletely understood problem. Immune mechanisms are often postulated when other explanations have been ruled out. Gammill and her colleagues ${ }^{14}$ report a 5 -year human clinical study designed to address whether naturally acquired microchimerism that a woman acquired from her own mother could be detected in women with recurrent miscarriage. Miscarriage was defined as three or more consecutive losses prior to week 20 of gestation. Differences in microchimerism versus control pregnancies were suggested. $\mathrm{Fu}$ and her colleagues ${ }^{15}$ continue the theme of recurring human pregnancy loss from the perspective of cellular immunity with an emphasis on TH17 cells. They focus on the need to create an appropriate niche in decidual tissue for correct regulatory cell interactions and propose the spectrum of outcomes from inappropriate TH17 regulation varies with preeclampsia being a less severe disturbance than pregnancy loss.

Another very common pathology of pregnancy is preterm labour. This problem is increasing in current societies and has major impacts on the health of the premature offspring through abrupt interruption of development. Gomez-Lopez and her colleagues ${ }^{16}$ summarize the current thinking regarding the contributions of both the adaptive and innate immune systems to normal labour and to preterm labour and highlight important differences between these two modes of delivery.
They thoroughly discuss changes in the decidua, myometrium, cervix, fetal membranes and fetal tissues and the interactions between immune cell subsets in women and in murine models.

The issues of maternal health and its impact on offspring do not end at birth. The peripartum interval (up to 28 days after birth) is one of great challenge for maintenance of newborn health with passive antibody transfer from the mother to fetus during gestation and from mother to child via lactation being of critical importance. Clark and colleagues ${ }^{17}$ report new studies on vaccination strategies to limit maternal infection by the intracellular bacterium Listeria monocytogenes with the hope of preventing fetal infection and death. Pathogen-specific CD8 ${ }^{+}$ $\mathrm{T}$ cells could be induced but were not protective in outbred matings, showing the complexity of many of the immunological issues that remain perplexing at the maternal-fetal interface.

In the final paper, Beaman and his colleagues ${ }^{18}$ look to the future and ask what will clinical laboratories do to support translation of basic findings in reproductive immunology to the patient care arena? They identify three areas in which patient workups will likely be incorporated to improve treatment for the prevention of abnormal pregnancies. These are enhanced semen analyses, endometrial growth factor measurements and natural killer cell assessments for early angiogenesis promotion in the placental bed. This contribution again emphasizes the importance of the immune system in supporting healthy pregnancies.

Together, the papers in this two issue miniseries give readers a broad overview of current research in reproductive immunology. They highlight normal basic studies and clinical aberrations of profound importance without claiming to cover the topic comprehensively. Many important questions remain unanswered and open for investigation. All of the contributing authors join me in inviting your experimental and reflective considerations of the issues raised in this miniseries and your building upon the current work we have collectively reported.

Kingston Ontario, Canada, 10 September, 2014

1 Nguyen PV, Kafka JK, Ferreira VH, Roth K, Kaushic C. Innate and adaptive immune responses in male and female reproductive tract in homeostasis and following HIV infection. Cell Mol Immunol 2014; 11: 410-427.

2 Matson BC, Caron KM. Mini Review: Adrnomedullin and endocrine control of immune cells during pregnancy. Cell Mol Immunol 2014; 11: 456-459.

3 Zhao S, Zhu W, Xue S, Han D. Testicular defense systems : immune privilege and innate immunity. Cell Mol Immunol 2014; 11: 428-437.

4 Du MR, Wang SC, Li DJ. The integrative roles of chemokines at the maternal-fetal interface in early pregnancy. Cell Mol Immunol 2014; 11: 438-448.

5 Lima PDA, Tu MM, Rahim MM, Peng AR, Croy BA, Makrigiannis AP. $L Y 49$ receptors activate angiogenic mouse $D B A^{+}$uterine natural killer cells. Cell Mol Immunol 2014; 11: 467-476.

6 Leno-Durán E, Munoz-Fernández R, Olivares EG, Tirado-González I. Natural killer cell and dendritic cell liasons in human gestation. Cell Mol Immunol 2014; 11: 449-455.

7 Rajagopalan S. HLA-G mediated NK cell senescence promotes vascular remodeling: implications for reproduction. Cell Mol Immunol 2014; 11: 460-466. 
8 Barker DJ, Thornburg KL. The obstetric origins of health for a lifetime. Clin Obstet Gynecol 2013; 56: 511-519.

9 Warren BD, Kinsey WK, McGinnis LK, Christenson LK, Kasto S, Stevens AM et al. Ovarian autoimmune disease: clincial concepts and animal models. Cell Mol Immunol 2014; 11: 510-521.

10 Lima PD, Zhang J, Dunk C, Lye SJ, Croy BA. Leukocyte driven-decidual angiogenesis in early pregnancy. Cell Mol Immunol 2014; 11: 522-537.

11 American College of Obstetricians and Gynecologists, Task Force on Hypertension in Pregnancy. Hypertension in pregnancy. Report of the American College of Obstetricians and Gynecologists' Task Force on Hypertension in Pregnancy. Obstet Gynecol 2013; 122: 1122-1131.

12 Bidarimath M, Khalaj K, Wessels JM, Tayade C. MicroRNAs, immune cells and pregnancy. Cell Mol Immunol 2014; 11: 538-547.

13 Tannetta DS, Dragovic RA, Alyahyaei Z, Southcombe JH. Extracellular vesicles and reproduction-promotion of successful pregnancy. Cell Mol Immunol 2014; 11: 548-563.
14 Gammill HS, Stephenson MD, Aydelotte TM, Nelson JL. Microchimerism in recurrent miscarriage. Cell Mol Immunol 2014; 11: 589-594.

15 Fu B, Tian Z, Wei H. TH17 cells $\mathrm{n}$ human recurrent pregnancy loss and pre-eclampsia. Cell Mol Immunol 2014; 11: 564-570.

16 Gomez-Lopez N, St Louis D, Lehr MA, Sanchez-Rodriguez EN, Areans-Hernandez M. Immune cells in term and preterm labor. Cell Mol Immunol 2014; 11: 571-581.

17 Clark DR, Chaturvedi V, Kinder JM, Jiang TT, Xin L, Ertelt JM et al. Perinatal Listeria monocytogenes susceptiblity despite preconceptural priming nad maintenance of pathogen-specific $\mathrm{CD}^{+}{ }^{\mathrm{T}}$ cells during pregnancy. Cell Mol Immunol 2014; 11: 595605.

18 Beaman KD, Jaiswal MK, Dambaeva S, Gilman-Sachs A. Future directions of clinical laboratory evaluation of pregnancy. Cell Mol Immunol 2014; 11: 582-588. 